properties. Sometimes it is not diagnosed by the available certified diagnostic preparations and test systems. In this regard, it is urgent to develop an effective method for identifying the pathogen using MALDI-TOF MS.

The aim is to study peculiarities of protein profiles of $\mathrm{Bru}$ cella $\mathrm{S}$ - and L-forms using mass spectrometric analysis.

The following Brucella strains of S- and L-forms were used in this study: B. abortus 544, B. melitensis $16 \mathrm{M}$, B. suis 1330, B. abortus I-206 of S- and L-forms, L-form of Brucella I-6, and L-form of Brucella I-7 from the collection of microorganisms of Irkutsk Antiplague Research Institute. Cultures were grown on Albimi agar at $37^{\circ} \mathrm{C}$ for 48 hours. Extraction was carried out with $70 \%$ formic acid followed by the addition of acetonitrile according to the "Instruction for Sample Preparation and Subsequent Mass Spectrometric Analysis of Pathogens of 1-3 Risk Groups" (Irkutsk, 2011). The spectra were collected using MicroFLEX mass spectrometer (Bruker Daltonics, Germany).

In the absence of Brucella spp. protein profiles in the database, identification of the pathogen did not provide reliable results. Therefore, during the first stage of the work the protein profiles of the following reference strains were added to the database: B. abortus 544, B. melitensis $16 \mathrm{M}$, and $B$. suis 1330 . Thereafter the mass spectrometric study of the other representatives of these three species allowed achieving the reliable identification to the species level except L-forms of B. abortus I-206. After the introduction of $B$. abortus $\mathrm{I}-206$ in $\mathrm{L}$-form into the database, it became possible to identify L-forms of this species, in particular L-forms of Brucella I-6 and Brucella I-7.

Based on the results, it can be assumed that L- and $\mathrm{S}$-forms of the same species differ significantly in protein profiles. Thus, we can recommend mass spectrometry with matrix-activated laser desorption/ionization for the accelerated identification of Brucella. For effective application of the method, it is necessary to create a representative electronic database of mass spectra of collection Brucella strains in both $\mathrm{S}$ - and L-forms.

\subsection{7 doi: 10.15789/2220-7619-2018-4-4.17 \\ COXIELLA BURNETII PREVALENCE IN TICKS \\ IN THE ULYANOVSK REGION}

Yu.A. Panferova ${ }^{1}$, O.A. Freylikhman ${ }^{1}$, N.K. Tokarevich ${ }^{1}$, A.A. Nafeev ${ }^{2}$, E.I. Sibaeva ${ }^{2}$

${ }^{1}$ St. Petersburg Pasteur Institute, St. Petersburg, Russia; ${ }^{2}$ Center for Hygiene and Epidemiology in the Ulyanovsk Region, Ulyanovsk, Russia

Q fever is on record in the Ulyanovsk Region, and 3 cases were reported in 2013-2017. (average annual incidence rate over the period is only 0.08 per 100000 ), but in fact the spread of $\mathrm{Q}$ fever is much higher judging by the results of seroprevalence survey in some districts where the antibodies to Coxiella burnetii were detected in $3.7 \%$ of healthy population. The role of ticks in the direct transmission of $C$. burnetii to humans is small, however, being important participants of the pathogen circulation in natural and mixed foci of the infection they pose a real threat to animals, including agricultural, that contribute much to $\mathrm{Q}$ fever outbreaks in humans. Hence, monitoring of $C$. burnetii in ticks is essential for $\mathrm{Q}$ fever prevention.

The study objective was to assess the $C$. burnetii prevalence in ticks and to conduct subsequent genetic analysis of PCR products.

709 adult ticks (Ixodes ricinus, Dermacentor marginatus, D. pictus, D. reticulatus) were flagged from vegetation in forest and forest-meadow sites in some districts of the
Ulyanovsk Region, and examined individually using standard PCR with the genus-specific primers flanking the $16 \mathrm{~S}$ ribosomal RNA gene site. For PCR-positive results the amplicons were sequenced.

Genetic markers of $C$. burnetii DNA were detected in 5 ticks (I. ricinus, D. marginatus, D. reticulatus) from the Cilninsky, Ulyanovsky, Melekessky, Kuzovatovsky and Novospassky districts. The homology of the nucleotide sequence of the 16S rRNA gene of four PCR products was 99\% as compared to the reference Nine Mile strain, while for one of them it was only $95 \%$, that justifies the need to further study the heterogeneity of the microorganisms of the genus Coxiella. One D. marginatus (Novospassky district) was possibly infected with a less-investigated coxiella-like microorganism

The existence of natural foci of Q fever was confirmed in 5 districts of the Ulyanovsk region. The genetic heterogeneity of $C$. burnetii circulating in the region was shown for the first time. The advisability of further study on the heterogeneity of microorganisms of the genus Coxiella is argued.

\subsection{8} doi: 10.15789/2220-7619-2018-4-4.18

\section{WHOLE GENOME-BASED PHYLOGENETIC DIVERSITY AND GENOMIC EPIDEMIOLOGY OF LEPTOSPIRA}

\section{Picardeau}

Institut Pasteur, Paris, France

Leptospirosis is an emerging zoonotic disease caused by pathogenic Leptospira strains. Each year, there is an estimated 1 million severe cases of leptospirosis and nearly 60000 deaths worldwide. The genus Leptospira is highly heterogeneous and currently consists of 23 species and more than 300 serovars that can be isolated from diverse ecological niches and animal reservoirs. According to their phylogeny, Leptospira species are distributed in 3 groups: the pathogens, the intermediate species, which cause a milder disease, and the saprophytes, which do not cause disease in human nor animals.

Different serological and molecular typing methods have been used to study the epidemiology of Leptospira, but they are performed by few reference laboratories and usually designed for the most commonly found pathogens. Since the first complete Leptospira genome sequence was published in 2003, it is now possible to sequence bacterial genomes in a few hours at reduced cost. Whole-genome sequencing (WGS) has emerged today as an ultimate tool for both the identification of relevant genetic variations linked to virulence and for bacterial strain typing.

In this study, the taxonomic status of all species of the genus Leptospira, as well as 81 strains isolated from the natural environment across a wide geographic range, was evaluated by comparative genomics. Our results reveal that the genus Leptospira now contains 65 named species, including species from a new sub-lineage. Our findings show that the genus has a large and open pan-genome which further confirms the complexity of this genus. The availability of whole-genome sequences of Leptospira also allowed us to develop a core genome MLST (cgMLST) scheme targeting the entire genus of Leptospira. Our cgMLST represents a standardized, accurate, highly discriminatory, and reproducible method for differentiation among Leptospira isolates, allowing for comparison of and sharing typing results among laboratories worldwide.

This study will advance many aspects of the leptospirosis field including epidemiology, diagnostics, and basic knowledge including species diversity, evolution, ecology, and virulence. 